\title{
Calidad del empleo de los adultos mayores en Chile, un factor de envejecimiento activo
}

\author{
Luz María Ferrada-Bórquez \\ Mauricio Alejandro Ferrada-Bórquez
}

\author{
Universidad de los Lagos, Chile \\ Ministerio del Trabajo y Previsión Social de Chile
}

Resumen

Se constata una creciente tasa de envejecimiento de la población acompañada de un aumento de la participación de adultos mayores en el mercado laboral de Chile. El propósito aquí es conocer cuales son sus condiciones de empleo. Para ello, se estiman indicadores parciales y sintéticos que develan la situación en los factores de seguridad social, contrato, jornada e ingresos laborales. Se obtienen diferencias significativas en los índices estimados entre hombres y mujeres, distintos niveles educativos, residencia geográfica, tamaño de empresa y sector económico en que trabajan. A su vez, son las condiciones de seguridad social y contrato laboral las más dispares. Estos resultados permiten argumentar elementos de política pública focalizadas en el contexto de un envejecimiento activo.

Palabras clave: Adulto mayor, envejecimiento activo, mercado laboral, envejecimiento de la población.

\section{Abstract}

\section{Employment quality of the elderly in Chile, an active aging factor}

It has been established that the aging rate of the population in Chile is growing, accompanied by the increased participation of senior citizens in the job market. The aim of this study is to determine the employment conditions of elderly people in Chile. To this end, partial and synthetic indicators are estimated, in order to reveal the situation regarding the following factors: social security, contractual arrangements, working hours and working income. Significant differences are obtained in the estimated indices between men and women, and according to educational level, geographical area of residence, company size and economic sector in which they work. In turn, the greatest levels of disparity were found in the conditions relating to social security and employment contract. These results allow us to argue in favor of targetted public policies in the context of a active aging.

Key words: Older adult, active aging, job market, aging population. 


\section{INTRODUCCIÓN}

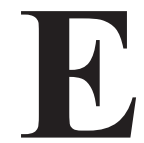

1 mundo occidental ha adoptado esquemas de desarrollo endógeno donde la ciencia y la técnica han sido condicionadas por la productividad, siendo el trabajo un eje central de este proceso. En esta lógica el recurso humano tiene valor económico y constituye un capital indispensable para el logro de los objetivos de las organizaciones. Ese formato permitiría tanto la acumulación de capital para el inversor, como la satisfacción de necesidades económicas al trabajador.

Sin embargo, este proceso ha estado acompañado por una dinámica caracterizada por un bajo crecimiento poblacional, derivado de menores tasas de fecundidad y mortalidad, aunque con un notable incremento de la esperanza de vida y longevidad. Las personas tienen mayor proyección de sobrevivencia y mejores condiciones físicas, lo que sucede tanto, en Europa, Estados Unidos como en toda la región de América Latina y el Caribe. Las estimaciones indican que para el año 2050, 22 por ciento de la población mundial corresponderá a adultos mayores (Olivi et al., 2015).

Lo anterior, ha llevado a la prolongación de la etapa laboral más allá de la jubilación, ya sea aumentando el número de años en la relación contractual con el empleador o mediante cambios de ocupación. La Figura 1 muestra antecedentes en cuanto a adultos mayores ocupados en Chile.

Se aprecia que la población de 60 años o más ha aumentado de manera importante; así entre los años 1990 y 2013 pasa de 1.31 a 2.89 millones de personas, representando al inicio del periodo 10.8 por ciento de la población chilena, y veintitrés años más tarde 16.7 por ciento.

A su vez, el porcentaje de ocupados se ha incrementado en todos los tramos de edad del segmento estudiado, y significativamente en las edades de 60 a 69 años.

La Organización Internacional del Trabajo (OIT) indica que los estados deben procurar el trabajo decente en todo el ciclo vital, incluida la vejez; a su vez, señala un conjunto de recomendaciones puntualizando:

...los trabajadores que por su edad están expuestos a afrontar dificultades para insertarse en el mercado laboral, estas recomendaciones abordan temas cruciales que hasta hoy son motivo de debate y puntos clave en la agenda pública: la igualdad de oportunidades y de trato, la protección en el empleo, el retiro, la utilización del tiempo libre, etc. (Paz, 2010, 22). 
Figura 1: Población de 60 años o más, Índice de envejecimiento $\left(^{*}\right)$ y porcentaje de ocupados en cada tramo de edad

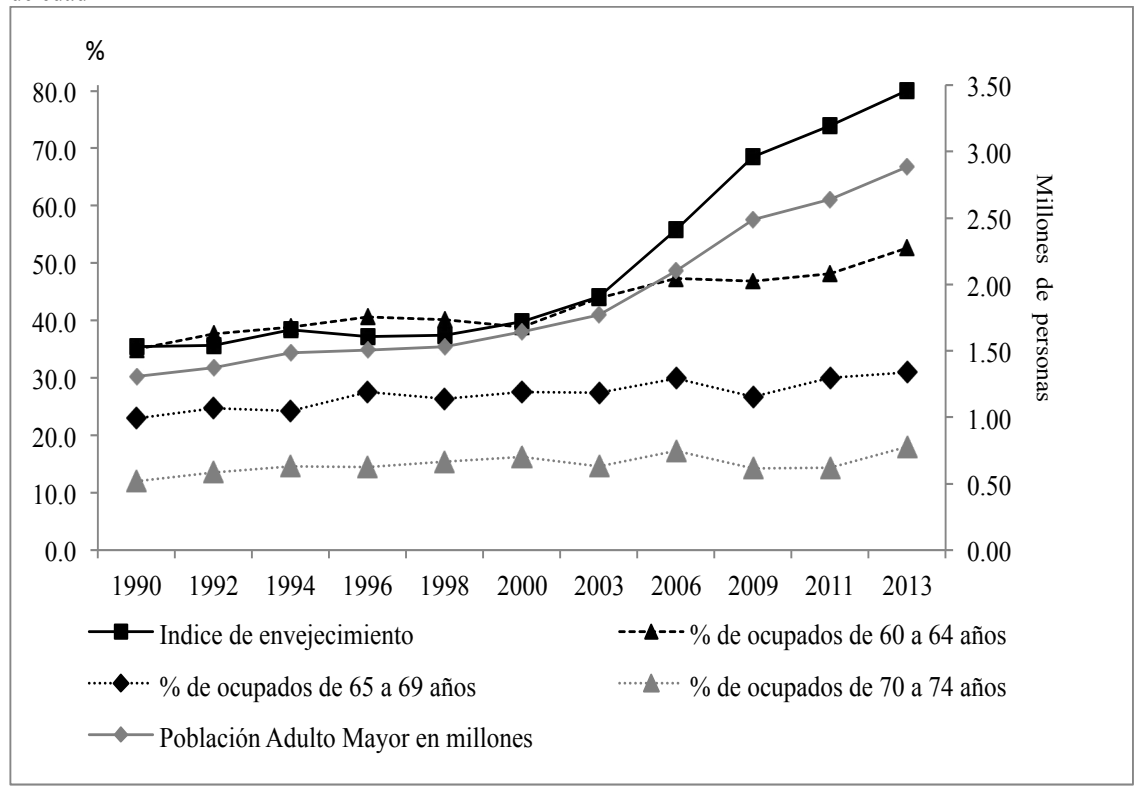

$\left({ }^{*}\right)$ Número de personas de 60 años o más por cada 100 menores de 15 años.

Fuente: elaboración propia, con base a las encuestas CASEN, de cada año.

En este contexto nos preguntamos ¿cómo son las condiciones de empleo en Chile para los trabajadores de 60 o más años de edad? La literatura especializada muestra algunos resultados respecto de la participación laboral, el ingreso y la situación contractual. En este caso se pretende profundizar en el conocimiento en cuanto a las condiciones de empleo de los adultos mayores en Chile, para lo cual se plantean los siguientes objetivos específicos: i) construir una medida de condiciones de empleo de los adultos mayores ii) estimar las condiciones de empleo de los adultos mayores en Chile y iii) comparar los resultados entre segmentos de la población.

Después de esta introducción, el trabajo desarrolla un apartado con los aspectos teóricos que fundamentan la importancia de esta temática, posteriormente se definen los aspectos metodológicos para responder la pregunta de investigación, luego se exponen los resultados para finalmente concluir.

\section{Aspectos Teóricos}

En esta investigación se emplean conceptos de la gerontología, disciplina científica que aborda las materias relacionadas con la vejez como etapa, y 
el envejecimiento como proceso, cuya rama social se encuentra dirigida a abordar los aspectos socioculturales, educativos y demográficos, y consta de dos componentes, la investigación y el trabajo comunitario con énfasis en la prevención de patologías psico-físicas. El propósito de esta acción disciplinar es lograr la prolongación de la vida (retardar la muerte), y mejorar la calidad de vida, lo cual implica que los adultos mayores deben contar con servicios que les permitan tener un acceso adecuado a las prestaciones de salud, educación, recreación y participación social (Pontificia Universidad Católica, 2012).

Los avances científicos han permitido, que al menos desde la academia no se asocie directamente a la vejez con el detrimiento en la salud, el aislamiento y/o la pérdida de habilidades sociales, señalándo que existe la posibilidad de un buen envejecer, vislumbrando metas en esta etapa del ciclo vital (Pontificia Universidad Católica, 2012).

Un enfoque teórico para abordar el estudio del envejecimiento es analizarlo a través de los factores psicosociales que impactan en la mantención de una vida activa con independencia, puesto que condicionan un envejecimiento exitoso (Fernández-García y Ponce de León-Romero, 2013). Desde fines de la década de 1990 se va desarrollando el concepto de envejecimiento activo. Al respecto la Organización Mundial de la Salud (OMS) propone adoptar este enfoque, considerando un conjunto de factores como la salud, la participación y la seguridad social. Dicho de otra forma, para que un persona logre su potencial de bienestar biológico, mental y social a lo largo de su vida, requiere que participe en forma continua en la sociedad. Así, se construye un nuevo paradigma, donde la vejez ya no es sinónimo de déficit sino de una etapa con posibilidades de desarrollo, optimización y compensación (Fernández-Ballesteros, 2012). Más tarde, se destacan a los factores económicos como uno de los determinantes del envejecimiento activo, incluyendo aquí al trabajo (OMS, 2002).

Para la optimización de las oportunidades, las políticas públicas dirigidas a este grupo etario deben procurar el cumplimiento de sus derechos sociales, económicos, culturales y de participación, en cuanto son seres humanos insertos en una comunidad política. Se trata de un cambio estratégico, en el cual las personas mayores pasan de ser consideradas sujetos pasivos, de caridad, ha sujetos activos demandantes de derechos inalienables.

Esta nueva óptica es el llamado enfoque de derechos, cuya perspectiva está centrada en el reconocimiento de las personas como titulares de derechos, lo que implica cambiar la forma de concebir, implementar y evaluar las políticas públicas "pasando de la percepción de personas con necesi- 
dades que deben ser asistidas, a sujetos con derecho a demandar ciertas prestaciones y acciones de parte del Estado y gobierno, así como a ser parte de su definición" (Ochoa, 2011).

Esto implica que el Estado asume el desafío de concretar políticas que consideren los derechos humanos universalmente reconocidos a todos los adultos mayores, reforzando la corresponsabilidad de los sujetos en la vigilancia de los mismos.

Es así como las Naciones Unidas establece normas universales que permiten el disfrute de las garantías básicas en la convivencia humana de este grupo etario, señalándo los principios de la independencia, la participación, los cuidados, la autorrealización y la dignidad (ONU, 1991).

La independencia de las personas guarda relación con la capacidad de decidir autónomamente sobre la forma de vivir la vida, el quehacer cotidiano y la implementación de las habilidades personales y colectivas, sin presión de ningún grupo social o familiar.

Los hombres y mujeres mayores tienen derecho al acceso a la alimentación, al agua, a la vivienda, al vestuario y a una atención de salud adecuada, mediante los ingresos obtenidos de su propia autosuficiencia, y del apoyo de su familia o de su comunidad. La forma de obtener ingresos se da mediante el acceso a pensiones una vez comenzada la jubilación, o mediante la oportunidad de ejercer el trabajo remunerado. En este sentido, son ellos quienes deben determinar el cuándo y en qué medida dejarán de desempeñar la actividad laboral.

Trabajar en forma asalariada o por emprendimiento (Oelckers, 2015) permite a las personas aprovechar las oportunidades para desarrollar sus potencialidades, sumar nuevas habilidades y construir una imagen personal positiva. De esta forma, la posibilidad de efectuar una labor pagada en esta etapa de la vida constituye una fuente de ingresos y una herramienta de realización personal, en la medida que el empleo ejercido sea de calidad.

Con el objetivo de medir esta categoría la OIT conceptualiza la noción de trabajo decente (OIT, 1999) definido como

la oportunidad de acceder a un empleo productivo que genere un ingreso justo, la seguridad en el lugar de trabajo y la protección social para las familias, mejores perspectivas de desarrollo personal e integración social, libertad para que los individuos expresen sus opiniones, se organicen y participen en las decisiones que afectan sus vidas, y la igualdad de oportunidades y trato para todos, mujeres y hombres (OIT, 2016).

Con base en esta clasificación se han efectuado numerosas investigaciones en el ámbito de las ciencias sociales y económicas, construyendo indi- 
cadores multivariados para evaluar las propiedades del empleo, en cuanto a la cuantificación y a la medición diacrónica del mismo.

En este artículo se responde a la pregunta de investigación señalada en la introducción, utilizando el enfoque de la OIT y las propuestas metodológicas Chacón (1999) y Farné (2003), ampliamente usada en trabajos para América Latina. Para el caso de Chile ha sido empleada por Alarcón y Santos (2008), Sehnbruch y Ruiz-Tagle (2010) y Cácerez y Zuñiga (2013); no obstante, a diferencia de ellos, en este caso será aplicada a la población de interés, por ello se realizan algunas adecuaciones. En el siguiente apartado se describe el método utilizado.

\section{Metodología Y DATOS}

La definición de calidad de empleo se basa en el trabajo de Chacón (1999: 218) que clasifica a los empleos como de buena calidad, baja calidad o precarios e intermedios, utilizando los factores de ingreso laboral, contrato laboral y seguridad social; más tarde, Ferné (2003) agrega la jornada laboral, calculado un índice parcial por factor, que finalmente sintetiza en un índice de calidad de empleo (ICE).

La idea de construir un indicador sintético implica resumir información muy diversa, en este caso agregar, tanto antecedentes monetarios como otras variables que impactan en la calidad de empleo de los adultos mayores; un propósito ciertamnete complejo, es por ello que nuestro objetivo es obtener una medida próxima de calidad de empleo, que además, permitan hacer comparaciones por segmento, en este caso por: sexo, zona urbano y rural, región, escolaridad, tamaño de empresa, rama de actividad económica y edad.

Siguiendo el trabajo de Ferné (2003), ello se logra obteniendo un indicador sintético por persona, a partir de ponderaciones horizontales y verticales. En el primer caso la idea es agregar los índices asociados a cada factor mediante ponderaciones fijas, así el autor propone 40, 25, 10 y 25 por ciento para los índices de ingreso (II), contrato laboral (ICL), jornada laboral (IJL) y seguridad social (ISS), respectivamente. Estas ponderaciones fueron validadas para Chile por Alarcón y Santos (2008). En definitiva ICE por persona se estima de la siguiente forma:

$$
\mathrm{ICE}_{\mathrm{i}}=0.4 * \mathrm{II}_{\mathrm{i}}+0.25 * \mathrm{ICL}_{\mathrm{i}}+0.25 * \mathrm{ISS}_{\mathrm{i}}+0.10 * \mathrm{IJL}_{\mathrm{i}} \ldots
$$


Siguiendo el enfoque señalado, las ponderaciones verticales son asignaciones de puntajes por trabajador, que indican cómo se encuentra en cada factor, y permitirán resolver el problema de distintas unidades de medidas de las variables (monetaria y no monetaria), y además, ser coherentes en cuanto a longitud y sentido de la escala; utilizando puntajes con un rango de 0 (empleo de baja calidad) a 100 (empleos de buena calidad).

En concreto, cada persona obtiene una puntuación según su condición en cada factor, que luego se resume en un índice sintético como se señala en la ecuación (1). En esta lógica, siguiendo a Ferné (2003) un trabajo será de baja calidad o precario cuando: sus ingresos son bajos, no tiene contrato laboral, trabaja menos de media jornada al mes y no tiene seguridad social, en dicho caso el puntaje ponderado tenderá a 0 ; por el contrario, el empleo es de buena calidad si se obtiene un ingreso superior, trabajo jornada completa, tiene contrato y seguridad social, por lo tanto, el puntaje se tendería a 100 .

Este estudio se realiza con base a la encuestas de Caracterización Socioeconómica Nacional (CASEN) en su última versión disponible, correspondiente al año 2013. Sin embargo, para ver la consistencia de los resultados se analizan también las de 2006 y 2000.

Así, de acuerdo con Ferné (2003) la ponderación vertical se define por factor, y permite generar índices por factor. Para este trabajo su contrucción se basará en la literatura especializada y las particularidades de la población estudiada, lo que se detalla a continuación:

\section{Índice del factor ingreso laboral (II)}

La metodología de Ferné (2003) sugiere asignar puntajes en relación al salario mínimo legal (SML), así se asigna una puntuación de 100 a aquellos que obtienen un ingreso superior a $2.3 \mathrm{SML}, 50$ si gana entre 2.3 y $1 \mathrm{SML}$ y 0 si recibe menos de $1 \mathrm{SML}$, este criterio es también es aplicado por Mora y Ulloa (2011) y Bustamante y Arroyo (2008), aunque en este último se exige más de tres SML para obtener 100 puntos y tener menos de 1.5 SML para calificar con 0 .

No obstante, en trabajos para Chile (Alarcón y Santos, 2008 y Cácerez y Zuñiga, 2013) el índice se estima en base al logaritmo natural del ingreso de la ocupación principal, estandarizándo con relación a su valor mínimo y máximo. Se calcula como:

$$
I I=\frac{\ln (\text { ingreso })-M \min \ln (\text { ingreso })}{\operatorname{Max} \ln (\text { ingreso })-\operatorname{Min} \ln (\text { ingreso })} * 100
$$


Esta opción que se utiliza en este trabajo, no sólo por su utilización anterior para el caso chileno, sino también porque ofrece una mayor variabilidad en el factor. De esta foma Max In (ingreso) representa el mayor ingreso expresado en logaritmo natural y Min ln (ingreso) el menor entre todos los sujetos de 60 años o más que trabajan ese año. Al multiplicar por $100 \mathrm{el}$ indicador queda expresado en un rango de 0 a 100.

\section{Índice del factor jornada laboral (IJL)}

Este factor se mide asignando un puntaje según el número de horas contratadas. Algunos autores señalan (Farné, 2003; Bustamante y Arroyo, 2008; Mora y Ulloa, 2011) dos alternativas: 100 puntos para aquellos trabajadores que laboran hasta 48 horas a la semana y 0 en caso si trabaja más; en cambio para el caso chileno (Alarcón y Santos, 2008; Cácerez y Zuñiga, 2013) se han definido tres categorías: 100 puntos para los que trabajan más de 35 horas a la semana hasta 50, en el caso de tener una jornada de 35 horas semanalas o menos 0 puntos, a su vez, si la jonada es mayor a 50 horas se le otorga 50 puntos.

Para este trabajo se sigue la lógica de los trabajos para Chile utilizando tres tramos; no obstante, dado que en el segmento estudiado trabajan en promedio menos horas a la semana que el total de los ocupados, la clasificasión se hará conforme a la jornada laboral legal (JLL) del sector privado, así el primer tramo corresponde a menos de media JLL, el tramo 2 al número de horas equivalentes a entre media y una JLL, y en el último cuando supera la JLL. La distribución de puntajes por encuesta sigue el siguiente patrón (Tabla 1):

Tabla 1: Puntaje del IJL para las encuestas Casen años 2013, 2006 y 2000

\begin{tabular}{crrrr}
\hline \multirow{2}{*}{ Tramos } & Casen 2013 & \multicolumn{3}{c}{ Casen 2006 y 2000 } \\
& Horas/semana & Puntaje & Horas/semanas & Puntaje \\
\hline 1 & Menor a 22 & 0 & Menor a 22 & 0 \\
2 & 22 a 45 & 100 & 22 a 48 & 100 \\
3 & Mayor a 45 & 50 & Mayor a 48 & 50 \\
\hline
\end{tabular}

Fuente: elaboración propia, con base a las encuestas CASEN, de cada año.

La diferencia entre Casen es porque en el periodo se registra una modificación legal en la jornada de trabajo chileno. El año 2000 y 2006 la jornada laboral de las empresas del sector privado era de 48 horas semanales, en cambio en 2013 es de 45 horas por semana. Sin embargo, en todo el periodo las empresas de sector público han tenido una jornada laboral 
de 44 horas semanales. A su vez, en la encuesta del año 2000 la pregunta pertinente consulta por la cantidad de horas mensuales de trabajo, a diferencia de las otras que preguntan por las horas semanales trabajadas, para corregirlo se ha supuesto un promedio 4.35 semanas por mes.

\section{Índice del factor contrato laboral (ICL)}

La literatura revisada define varias opciones que va desde el trabajador que tiene contrato permenente (en otros trabajos se menciona como indefinido) en cuyo caso se asignan 100 puntos, a no tener contrato se asigna un puntaje 0 , a su vez, si tuvierese un contrato distinto a permanenete (temporal, fijo u otro) definen 50 puntos (Farné, 2003; Alarcón y Santos, 2008; Bustamante y Arroyo, 2008; Mora y Ulloa, 2011 y Cácerez y Zuñiga, 2013). Para este estudio el índice se contruye en función a si tiene o no contrato laboral escrito y si es de tipo permanente o no, de manera que si en la encuesta indica que ha firmado contrato y es permanate se asigna un puntaje de 100, si señala tener sólo contrato escrito o permanente pero no ha fimado contrato obtiene un puntaje 50, indica un trabajo no permanate, sin contrato o no lo señala, su puntaje será 0 .

\section{Índice del seguridad social (ISS)}

Para este factor todos los trabajos señalados usan la misma tipificación, por lo que construirán las categorías de la misma forma, estos es, si el trabajador cotiza tanto para previsión social como salud, en cuyo caso se asigna un puntaje de 100; si cotiza sólo en uno se evalúa con 50; y si no lo hace con 0 .

De esta forma se obtiene una puntuación por persona en cada factor, que expresa su situación en cuanto a cada factor indicado en una misma escala.

Por lo tanto, lo que se realizará es la aplicación de una metodología conocida y validada, tanto para Chile como otros países, con algunas precisiones ya explicadas, conforme a las características de la poblacion estudiada y la disponibilidad de información.

Finalmente, es posible estimar un ICE por persona siguiendo la ecuación (1), y a su vez, analizar el resultado del indicador en distintos segmentos de la población, en los tres años de estudio. Para ello, se calcula la media del ICE entre las observaciones que componen cada tramo:

$$
I C E_{s}=\frac{1}{N_{s}} \sum I C E_{i} \ldots \ldots \ldots
$$


Siendo ICEs la calidad del empleo de los $\mathrm{N}$ trabajadores que se ubican en el segmento $s$.

Así, las estimaciones permiten conocer la posición relativa que tienen los adultos mayores en calidad del empleo a nivel global y en cada uno de los factores, por segmento.

Al finalizar, se evalúa si hay deferencias significativas entre los segmentos. Para ello, se utiliza la prueba de medianas de Kruskal Wallis (KW) que contrasta la hipótesis nula de que $k$ muestras obtenidas pertenecen a una misma población. Se elige este test estadístico pues a diferencia del t-student no se exige homocedasticidad ni la normalidad en la distribución de datos.

\section{ANÁlisis y Resultados}

Como se ha indicado, se estima un ICE donde el segmento está referido a las variables sexo, zona geográfica, región, escolaridad, tamaño de la empresa, rama de actividad económica y edad. De esta forma, se analiza la posición relativa de cada segmento respecto del indicador en cada año. Los resultados se exponen en la Tabla 2 y en la Tabla 3.

Éstos constituyen un insumo pertinente para hacer análisis del comportamiento del mercado de trabajo para proponer algunas medidas desde la política pública que permitan mejorar las condiciones laborales de la población adulta mayor.

En primer lugar se examina el indicador para toda la población en condición ocupada, distribuída por tramo de edad. Posteriormente, el análisis se concentrará en la mediciones factoriales y global para las personas que están en el tramo de edad de estudio (60 o más años), con el objeto de indagar en la situación de la calidad del empleo.

La evidencia indica (Figura 2) que los adultos mayores registran menor calidad de empleo que los jovenes adultos. Sin embargo, los más jóvenes, entre 15 y 24 años, exiben una situación inferior debido principalmente a déficit en seguridad social. No obstante, los mayores de 65 años obtiene las peores condiciones en el indicador global.

Sorprende que el II (Figura 2) sea muy similar entre los tramos de edad; es problable que sea consecuencia de calcular el índice mediante la aplicación del logaritmo natural de ingreso, lo que suavisa las variaciones. 
Tabla 2: Recuento y valor medio del ICE por región, tramo de edad, escolaridad, rama, tamaño de empresa, sexo y zona geográfica

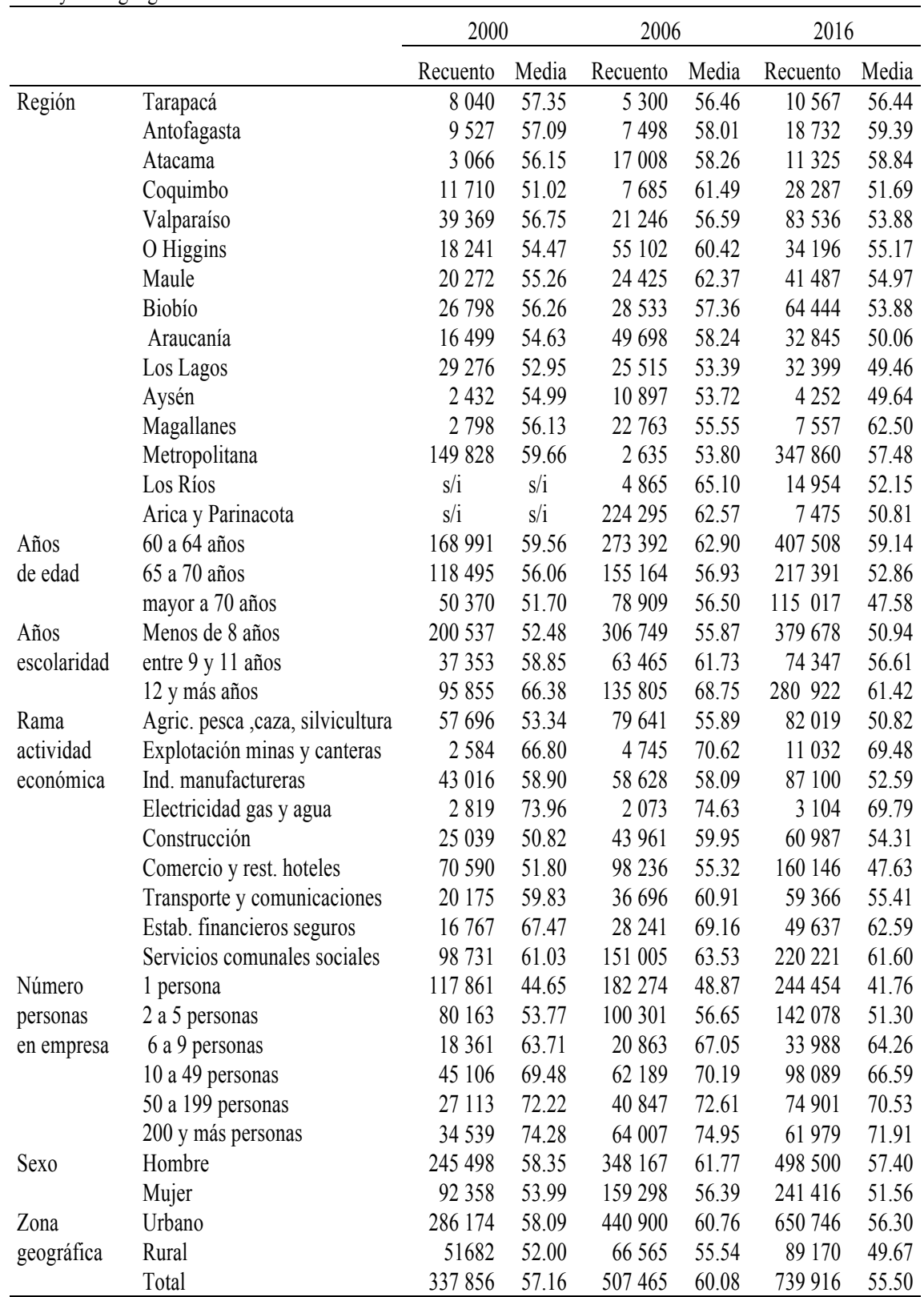

s/i: sin información, en los 2000 y 2006 señalados, las regiones de Los Ríos y Arica, forman parte de las regiones de Los Lagos y

Tarapacá, respectivamente. 


\begin{tabular}{|c|c|c|c|c|c|c|c|c|c|c|c|c|c|c|c|c|}
\hline $\bar{\pi}$ & 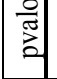 & $\begin{array}{l}* \\
* \\
*\end{array}$ & * & $\begin{array}{l}* \\
* \\
*\end{array}$ & $\begin{array}{c}* \\
* \\
*\end{array}$ & $\begin{array}{l}* \\
* \\
*\end{array}$ & * & $\begin{array}{l}* \\
* \\
*\end{array}$ & $\begin{array}{l}* \\
* \\
*\end{array}$ & $\begin{array}{l}* \\
* \\
*\end{array}$ & $\stackrel{*}{*}$ & $\begin{array}{l}* \\
* \\
*\end{array}$ & * & $\stackrel{*}{*}$ & $\begin{array}{l}* \\
* \\
*\end{array}$ & * \\
\hline 至 & $x$ & $\begin{array}{c}\vec{b} \\
\stackrel{n}{d}\end{array}$ & 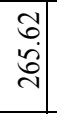 & $\stackrel{9}{8}$ & \begin{tabular}{l|}
0 \\
0 \\
0 \\
$\dot{1}$ \\
\end{tabular} & 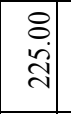 & $\begin{array}{c}\tilde{\delta} \\
\tilde{g}\end{array}$ & $\begin{array}{l}\text { गे } \\
\stackrel{2}{2}\end{array}$ & $\begin{array}{c}\hat{\delta} \\
\tilde{n} \\
\sim \\
\end{array}$ & $\begin{array}{l}\tilde{\hat{T}} \\
\dot{\tau} \\
\end{array}$ & $\begin{array}{c}\infty \\
\stackrel{\infty}{m} \\
\dot{m}\end{array}$ & $\begin{array}{l}\tilde{\alpha} \\
\tilde{\alpha}\end{array}$ & $\begin{array}{l}\infty \\
\infty \\
i n \\
i n \\
\end{array}$ & $\begin{array}{l}\infty \\
? \\
0 \\
0 \\
-1 \\
\end{array}$ & $\begin{array}{l}\Omega \\
\cong \\
\\
\end{array}$ & $\begin{array}{l}\vec{b} \\
\tilde{m} \\
m\end{array}$ \\
\hline 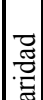 & \begin{tabular}{|l|}
$\frac{0}{\pi}$ \\
$\frac{\pi}{2}$
\end{tabular} & $\begin{array}{l}* \\
* \\
*\end{array}$ & $\begin{array}{l}* \\
* \\
*\end{array}$ & $\begin{array}{l}* \\
* \\
*\end{array}$ & $\begin{array}{l}* \\
* \\
*\end{array}$ & $\begin{array}{l}* \\
* \\
* \\
*\end{array}$ & $\begin{array}{l}* \\
* \\
*\end{array}$ & $\begin{array}{l}* \\
* \\
*\end{array}$ & $\begin{array}{l}* \\
* \\
*\end{array}$ & & $\begin{array}{l}* \\
* \\
*\end{array}$ & $\begin{array}{l}* \\
* \\
*\end{array}$ & * & $\begin{array}{l}* \\
* \\
*\end{array}$ & * & $*$ \\
\hline$\left|\begin{array}{|c}\pi \\
0 \\
0 \\
w 1 \\
1\end{array}\right|$ & $x$ & $\begin{array}{l}\stackrel{2}{0} \\
\grave{0} \\
2\end{array}$ & $\begin{array}{l}n \\
\grave{i} \\
\stackrel{m}{2}\end{array}$ & $\begin{array}{l}\stackrel{\infty}{\infty} \\
\infty\end{array}$ & 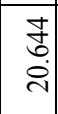 & $\begin{array}{l}\stackrel{a}{2} \\
\hat{n} \\
\end{array}$ & 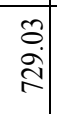 & 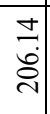 & $\begin{array}{c}0 \\
\stackrel{0}{0} \\
\infty \\
-\infty\end{array}$ & $\begin{array}{c}\stackrel{ \pm}{n} \\
\stackrel{n}{-}\end{array}$ & 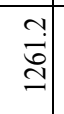 & $\begin{array}{l}\vec{m} \\
\dot{8} \\
\dot{q}\end{array}$ & $\begin{array}{l}0 \\
0 \\
\dot{\eta} \\
=\end{array}$ & $\begin{array}{c}a \\
\vec{n} \\
=\end{array}$ & $\begin{array}{l}\infty \\
\infty \\
\dot{ \pm} \\
+\end{array}$ & $\stackrel{\overbrace{}}{\overparen{\sigma}}$ \\
\hline$\Xi$ & \begin{tabular}{|l|}
$\frac{5}{\pi}$ \\
$\frac{\pi}{2}$ \\
\end{tabular} & $\begin{array}{l}* \\
* \\
*\end{array}$ & $\begin{array}{l}* \\
* \\
*\end{array}$ & $\begin{array}{l}* \\
* \\
*\end{array}$ & $\begin{array}{l}* \\
* \\
* \\
*\end{array}$ & 荬 & $\begin{array}{l}* \\
* \\
*\end{array}$ & $\begin{array}{l}* \\
* \\
*\end{array}$ & $\begin{array}{l}* \\
* \\
*\end{array}$ & $\begin{array}{l}* \\
* \\
*\end{array}$ & $\underset{*}{*}$ & $\begin{array}{l}* \\
* \\
*\end{array}$ & $\begin{array}{l}* \\
* \\
*\end{array}$ & $\stackrel{*}{*}$ & $\begin{array}{l}* \\
* \\
*\end{array}$ & $\begin{array}{l}* \\
* \\
*\end{array}$ \\
\hline 胥 & $\approx$ & 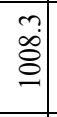 & 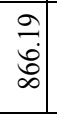 & $\begin{array}{l}\text { aे } \\
\text { d. }\end{array}$ & $\begin{array}{l}0 \\
0 \\
0 \\
-0 \\
\end{array}$ & 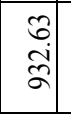 & $\begin{array}{l}m \\
\stackrel{9}{+} \\
\end{array}$ & $\begin{array}{l}\hat{a} \\
\dot{b} \\
n \\
n\end{array}$ & 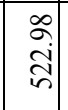 & 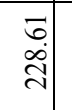 & 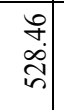 & \begin{tabular}{l}
$\vec{d}$ \\
\multirow{2}{*}{} \\
$\tilde{m}$
\end{tabular} & $\begin{array}{l} \\
\grave{d} \\
\text { in }\end{array}$ & $\begin{array}{c}2 \\
\vdots \\
i \\
5 \\
i n\end{array}$ & $\begin{array}{l}\overline{7} \\
\stackrel{2}{2} \\
\stackrel{2}{r}\end{array}$ & $\begin{array}{l}\mathcal{I} \\
\stackrel{J}{J} \\
\end{array}$ \\
\hline & 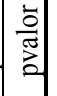 & $\begin{array}{l}* \\
* \\
*\end{array}$ & $\begin{array}{l}* \\
* \\
*\end{array}$ & $\begin{array}{l}* \\
* \\
*\end{array}$ & $\begin{array}{l}* \\
* \\
*\end{array}$ & $\begin{array}{c}* \\
* \\
*\end{array}$ & $\begin{array}{l}* \\
* \\
*\end{array}$ & $\begin{array}{l}* \\
* \\
*\end{array}$ & $\begin{array}{l}* \\
* \\
*\end{array}$ & $\begin{array}{c}* \\
* \\
* \\
*\end{array}$ & $\begin{array}{l}* \\
* \\
*\end{array}$ & $\begin{array}{l}* \\
* \\
*\end{array}$ & $\begin{array}{l}* \\
* \\
*\end{array}$ & $\begin{array}{l}* \\
* \\
*\end{array}$ & * & * \\
\hline 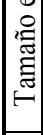 & $\approx$ & 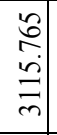 & $\stackrel{\mathbb{N}}{\mathbf{N}}$ & $\begin{array}{l}\infty \\
\vdots \\
\vdots \\
\vdots \\
1\end{array}$ & 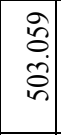 & $\begin{array}{l}\mathbf{E} \\
0 \\
0 \\
0 \\
-\end{array}$ & $\begin{array}{l}\vec{m} \\
\stackrel{\hat{n}}{\hat{n}} \\
\end{array}$ & 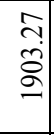 & \begin{tabular}{|c|c}
$n$ & $n$ \\
0 \\
0 \\
$\infty$ \\
$=$ \\
$=$
\end{tabular} & $\begin{array}{c}f \\
+ \\
+ \\
\dot{0} \\
n\end{array}$ & $\begin{array}{l}\text { } \\
\stackrel{ి}{\hat{~}}\end{array}$ & $\begin{array}{l}n \\
\vdots \\
\vdots \\
0 \\
0 \\
-1\end{array}$ & 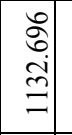 & $\begin{array}{l}\widehat{\infty} \\
\infty \\
\mathbb{S} \\
\tilde{\Xi} \\
\end{array}$ & $\begin{array}{l}0 \\
\stackrel{2}{a} \\
\stackrel{8}{0}\end{array}$ & 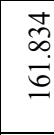 \\
\hline$\%$ & 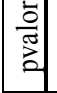 & $\begin{array}{l}* \\
* \\
*\end{array}$ & $\begin{array}{l}* * \\
* \\
*\end{array}$ & $\begin{array}{l}* \\
* \\
*\end{array}$ & * & * & $\begin{array}{l}* \\
* \\
*\end{array}$ & $\begin{array}{l}* \\
* \\
*\end{array}$ & $\begin{array}{l}* \\
* \\
*\end{array}$ & * & $\begin{array}{l}* * \\
*\end{array}$ & $\begin{array}{l}* \\
* \\
*\end{array}$ & 萰 & $\stackrel{*}{*}$ & * & * $*$ \\
\hline$\tilde{n}$ & $x$ & $\begin{array}{l} \\
\stackrel{9}{9}\end{array}$ & $\begin{array}{c}\stackrel{a}{1} \\
\dot{1}\end{array}$ & $\begin{array}{l} \\
\stackrel{0}{2} \\
\stackrel{2}{7}\end{array}$ & 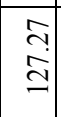 & $\begin{array}{c}7 \\
0 \\
0 \\
m\end{array}$ & 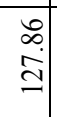 & $\begin{array}{l}\stackrel{+}{\circ} \\
\stackrel{\leftrightarrow}{\leftarrow}\end{array}$ & $\begin{array}{l} \\
\dot{0} \\
\vdots\end{array}$ & 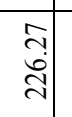 & $\begin{array}{c}\widehat{\infty} \\
\vec{b} \\
-\end{array}$ & 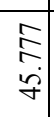 & $\left|\begin{array}{l}\mathfrak{a} \\
\widehat{b} \\
-\end{array}\right|$ & $\begin{array}{l}\overleftarrow{S} \\
\stackrel{J}{ \pm}\end{array}$ & $\begin{array}{l}n \\
0 \\
0 \\
b\end{array}$ & $\begin{array}{l}\infty \\
\stackrel{0}{2} \\
2 \\
\tilde{m}\end{array}$ \\
\hline & \begin{tabular}{|l|} 
\\
aे \\
aे \\
\end{tabular} & $\begin{array}{l}* \\
* \\
*\end{array}$ & * & $\begin{array}{l}* \\
* \\
*\end{array}$ & & $\begin{array}{l}* \\
* \\
*\end{array}$ & $\begin{array}{l}* \\
* \\
*\end{array}$ & $\begin{array}{l}* \\
* \\
*\end{array}$ & * & $\begin{array}{c}* \\
* \\
*\end{array}$ & $\stackrel{*}{*} \underset{*}{*}$ & $\begin{array}{l}* \\
* \\
*\end{array}$ & * & $\stackrel{*}{*}$ & & * \\
\hline$\stackrel{2}{N}$ & $\approx$ & 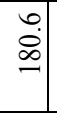 & $\begin{array}{c}\tilde{r} \\
\hat{o} \\
\dot{0}\end{array}$ & $\begin{array}{l}\infty \\
\dot{0} \\
0 \\
0\end{array}$ & $\begin{array}{l}\text { ț } \\
\text { ?. } \\
0\end{array}$ & 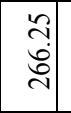 & $\begin{array}{l}\hat{\widehat{T}} \\
\stackrel{\infty}{0} \\
\end{array}$ & $\begin{array}{l}\widehat{n} \\
\tilde{n} \\
\\
n\end{array}$ & 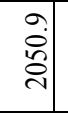 & $\begin{array}{l}\overrightarrow{3} \\
\text { î } \\
\end{array}$ & 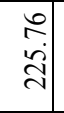 & $\begin{array}{l}0 \\
\grave{d} \\
\end{array}$ & \begin{tabular}{|c|}
0 \\
0 \\
0 \\
0 \\
\end{tabular} & $\begin{array}{l}0 \\
+ \\
5 \\
n\end{array}$ & $\begin{array}{l}\vec{b} \\
\dot{0}\end{array}$ & $\stackrel{\circ}{\stackrel{2}{0}}$ \\
\hline & \begin{tabular}{|l} 
离 \\
है
\end{tabular} & $\begin{array}{l}* \\
* \\
*\end{array}$ & * & * & $\begin{array}{l}* \\
* \\
*\end{array}$ & $\begin{array}{l}* \\
* \\
*\end{array}$ & $\begin{array}{l}* \\
* \\
*\end{array}$ & $\begin{array}{l}* \\
* \\
*\end{array}$ & * & $\begin{array}{l}* \\
* \\
*\end{array}$ & * & $\begin{array}{l}* \\
* \\
*\end{array}$ & * & $\begin{array}{l}* \\
* \\
*\end{array}$ & $\begin{array}{l}* \\
* \\
*\end{array}$ & * \\
\hline 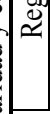 & $\nexists$ & $\begin{array}{c}m \\
\dot{\vec{D}}\end{array}$ & $\underset{\substack{0 \\
m}}{\stackrel{m}{m}}$ & 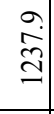 & 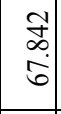 & $\begin{array}{l}\vec{n} \\
\tilde{n} \\
n\end{array}$ & 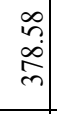 & $\begin{array}{c}n \\
\dot{J} \\
-\end{array}$ & $\begin{array}{c}r \\
\infty \\
0 \\
\sim\end{array}$ & $\begin{array}{l}0 \\
0 \\
\dot{0} \\
-\end{array}$ & $\begin{array}{c}\stackrel{n}{a} \\
\dot{i g} \\
y\end{array}$ & $\begin{array}{l}0 \\
\stackrel{0}{a} \\
\hat{v}\end{array}$ & $\begin{array}{l}\hat{0} \\
\mathbf{I} \\
-\end{array}$ & $\begin{array}{c}\infty \\
\infty \\
\infty \\
\Xi \\
- \\
-\end{array}$ & 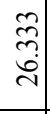 & $\vec{i}$ \\
\hline & & $\underline{\underline{U}}$ & 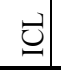 & $\begin{array}{l}\tilde{2} \\
\cong\end{array}$ & $\Xi$ & $=$ & 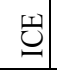 & $\vec{己}$ & $\begin{array}{l}\approx \\
\approx\end{array}$ & $\Xi$ & $=$ & 焉 & $\overrightarrow{0}$ & $\approx$ & 目 & $=$ \\
\hline & & & & $\overline{\mathrm{n}}$ & & & & & ષ্ণ & & & & & ষ্ণ & & \\
\hline
\end{tabular}


Figura 2: ICE, ICL, ISS, IJL, II, por tramo de edad, con base a CASEN 2013

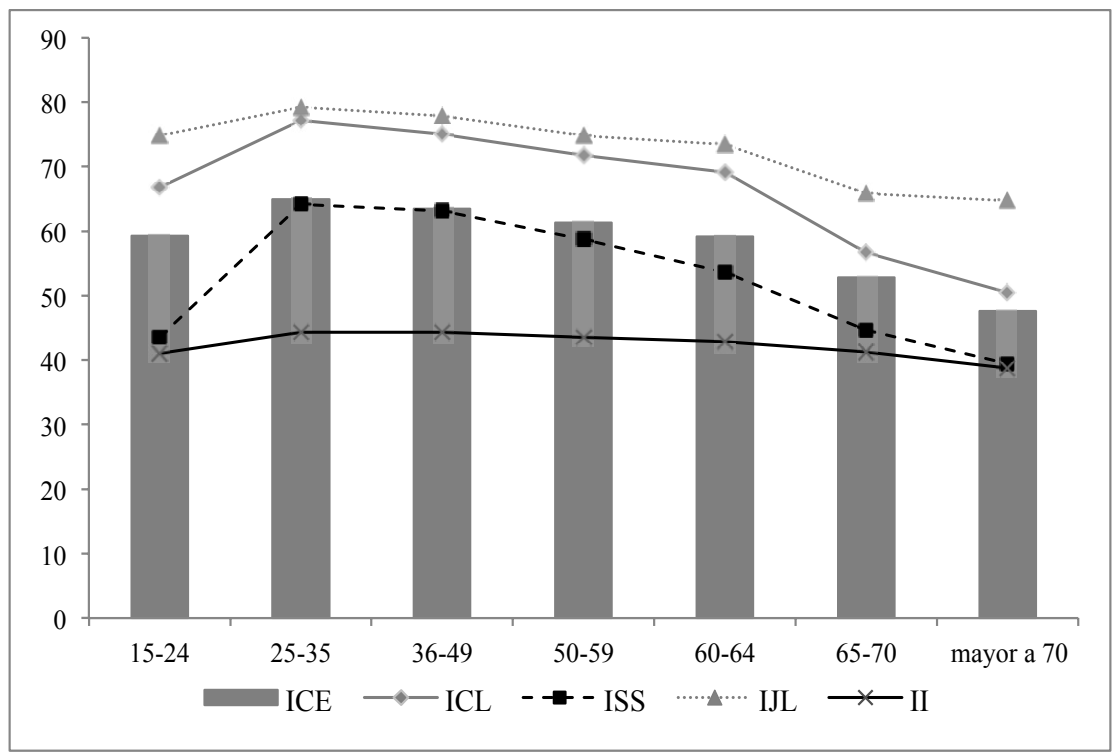

Fuente: elaboración propia.

\section{Calidad de empleo de adultos mayores en distintos Segmentos}

En lo que sigue se analiza la relación en los indicadores y los segmentos indicados. Las variables para segmentar se justifican en la literatura especializada, como se verá en este apartado.

Con relación a la zona, los investigadores señalan que la participación laboral de los adultos mayores en la zona rural es mayor, aún cuando los salarios son inferiores. Sin embargo, se indica que las mujeres adultas mayores que trabajan en la zona urbana duplican a las de la zona rural, aunque esto se debería a que las mujeres en la ruralidad tienden a ejercer labores no declaradas e incluso por intercambio de bienes (Marfán, 2007).

Las estimaciones (Figura 3) permiten concluir que los trabajadores que residen en el sector rural poseen un menor ICE, y que los índices con mayores brechas son ICL e ISS. Probabemente ello se debe a las características del trabajo en el sector rural, muchos de ellos se desarrollan labores estacionales, en tales condiciones, el contrato por lo general es temporal o no existe y en muy poca ocasiones se cuenta con seguridad social.

Ahora bien, los niveles de capital humano debiesen estar asociados con calidad de empleo, de hecho los estudios señalan que la educación formal está asociada, tanto a mayores ingresos laborales como la posibilidad tener 
un contrato laboral escrito y permanente, poseer seguridad laboral y permanecer en la jornada laboral legal.

Figura 3: Indicadores de calidad de empleo por zona urbano-rural

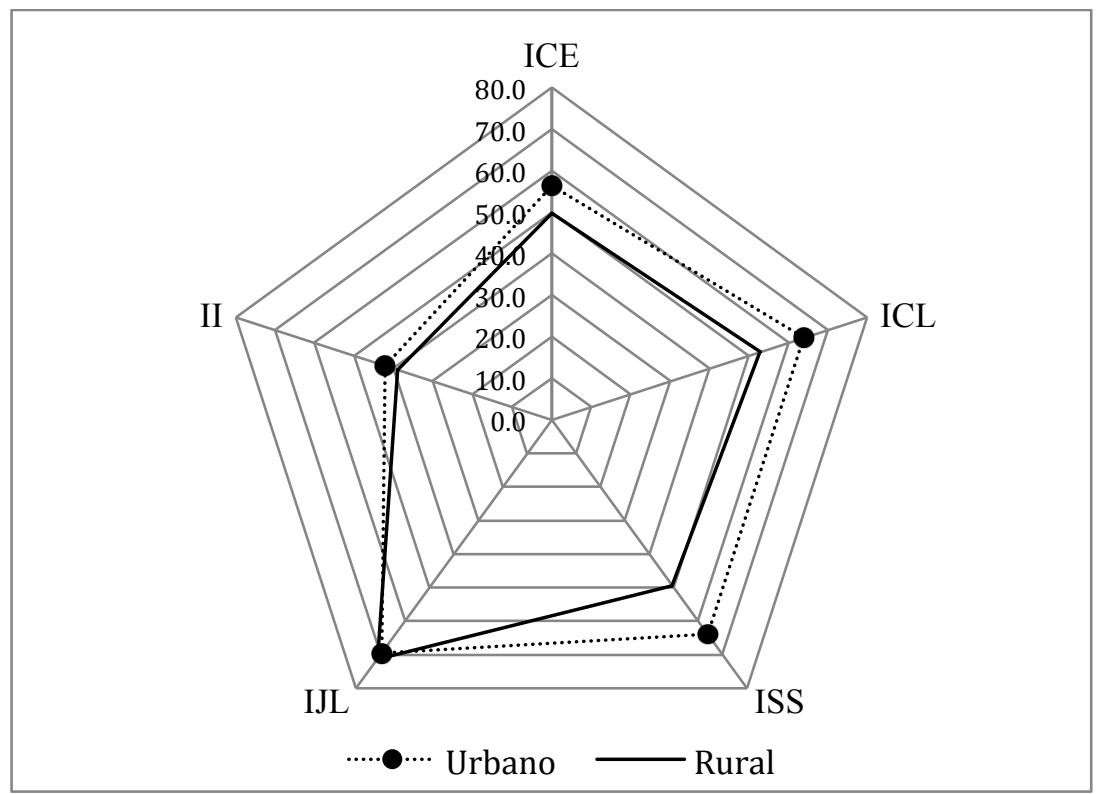

Fuente: elaboración propia.

Además, diferentes estudios indican que los adultos mayores con menor educación tienden al retiro laboral a la edad legal de jubilación, en contraste a los que cuentan con más escolaridad pues mantienen más tiempo trabajando más allá de los 65 años, a su vez "hay una tendencia a que los trabajadores menos educados y los que se emplean en trabajos de menor calificación, sean los más propensos a quedar desempleados y a convertirse en inactivos (Marfán, 2007).

Para este caso, con base a la distribución de frecuencia de la variable años de escolaridad, la población objeto de estudio se divide en tres tramos según edad. Se obtiene que aquellos con escolaridad igual a ocho años o menos son los que observan menor calidad de empleo, al contrario los con mayores credenciales educativas (12 años de escolaridad o más) obtienen empleo de mayor calidad, en particular en contratos y seguridad social (Figura 4). También, se aprecia que es el ISS el que tiene mayor distancia entre los más y menos educados. 
Figura 4: Indicadores de calidad de empleo por años de escolaridad en tramos

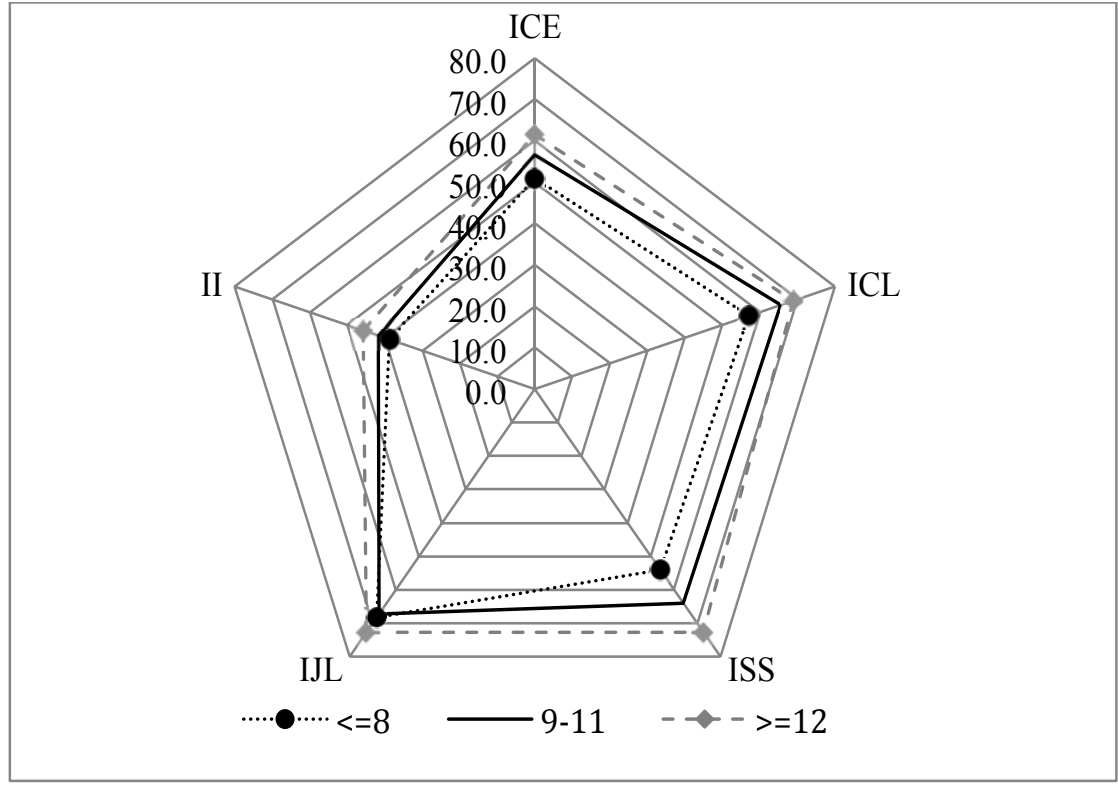

Fuente: elaboración propia.

Otro aspecto ampliamente señalado por la literatura especializada son las diferencias tanto en participación laboral como en los ingreso laborales, observando una ventaja para los varones. Los autores señalan que pesar del aumento de la participación femenina en el mercado laboral y de buenos indicadores de educación en ellas, persisten diferencias entre sexo. Contreras y Plaza (2007) indican que la existencia de ciertos patrones culturales explicaría gran parte de la baja participación femenina en Chile. Para el caso de los índices calculados se confirman dichas conclusiones (Figura 5), los hombres observan un mayor II y a la vez una mayor CE global (ICE); no obstante, las disparidades se incrementan en la jornada laboral (IJL) y seguridad social (ISS).

Por otro lado, los datos señalan que los trabajadores de empresas pequeñas en promedio reciben ingresos más bajos. A su vez, las unidades productivas de mayor tamaño (de 200 y más personas) presentarían mayores índices de protección en la seguridad social a los trabajadores mayores, "éstas protegen contra contingencias económicas y sociales provenientes del desempleo, o de otras tales como la invalidez o la muerte" (Paz, 2010). Para efecto de este estudio se utiliza el número de trabajadores (variables 
indicativa del tamaño de la organización) en tramos según identifica la encuesta CASEN, lo que coincide con los rangos que usa la OIT en otros estudios para el caso chileno.

Figura 5: Indicadores de calidad de empleo por sexo

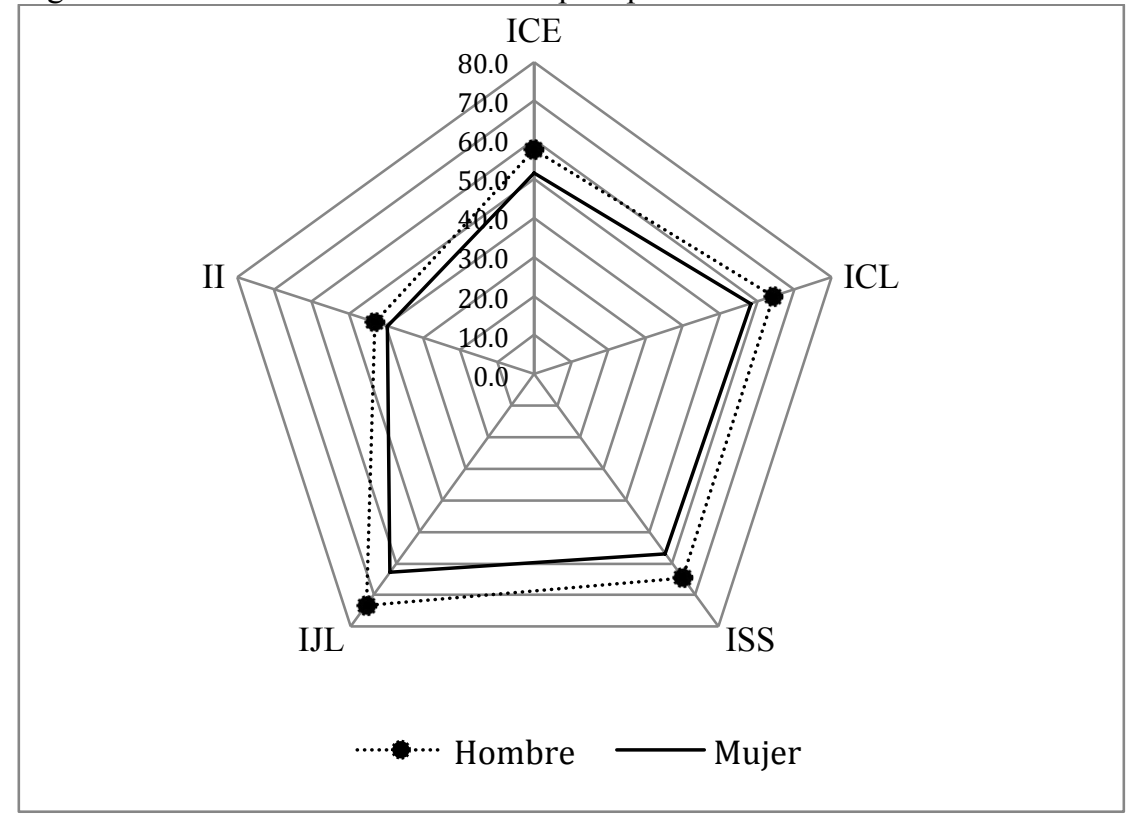

Fuente: elaboración propia.

A partir del Figura 6 se concluye que efectivamente los menores ICE están en los trabajadores de empresas menores de 10 trabajadores, y que los ICL e ISS son sustancialmente más bajos en la microempresa (de nueve o menos trabajadores). A diferencia de las conclusiones de la literatura en este caso se obtiene que entre las empresas grandes y medianas (de $50 \mathrm{o}$ más empleos) no hay deferencias sustantivas (Figura 6).

De esta forma se confirma que los adultos mayores con mejor ICE son aquellos con altas credenciales educativas, principalmente hombres, que habitan en el sector urbano y que laboran en empresas medianas y grandes. Dichos antecedentes se corroboran a su vez para los años 2006 y 2000 (Tabla 2). 
Figura 6: Indicadores de calidad de empleo por tamaño de empresa

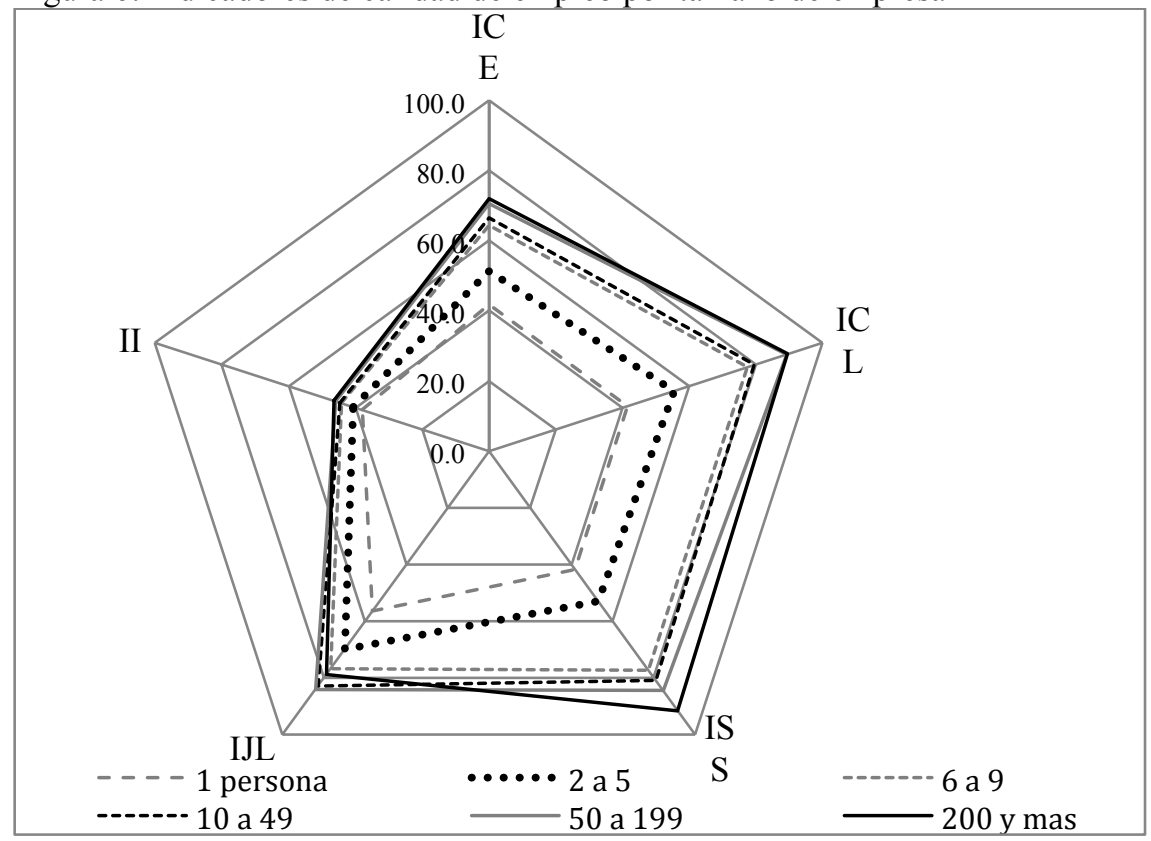

Fuente: elaboración propia.

\section{ICE Y REGIONES}

En la Tabla 4 se ha ordenado a las regiones chilenas según los resultados del ICE, a su vez se agregan el ICE obtenido por región y año, y los indices en cada factor. Se debe considerar que si bien no es posible comparar los resultados entre los años en términos absolutos, si en témirmos relativos y es lo que se realiza en este punto.

Se observa, que en 2013 la región de Magallanes, Antogasta, Atacama y Metropolitana son las que exhiben mayor calidad de empleo; en el extremo contrario las regiones de Araucania, los Lagos y Aysén obtienen un peor índice. Probablemente, este resutado este impactado fuertemente por el factor ingreso, pues es el factor que se incorpora al indicador sintético con mayor ponderación. No obstante, observando los indicadores por factor, se concluye que las mejores posiciones están influídas también por el ICL y ISS. 


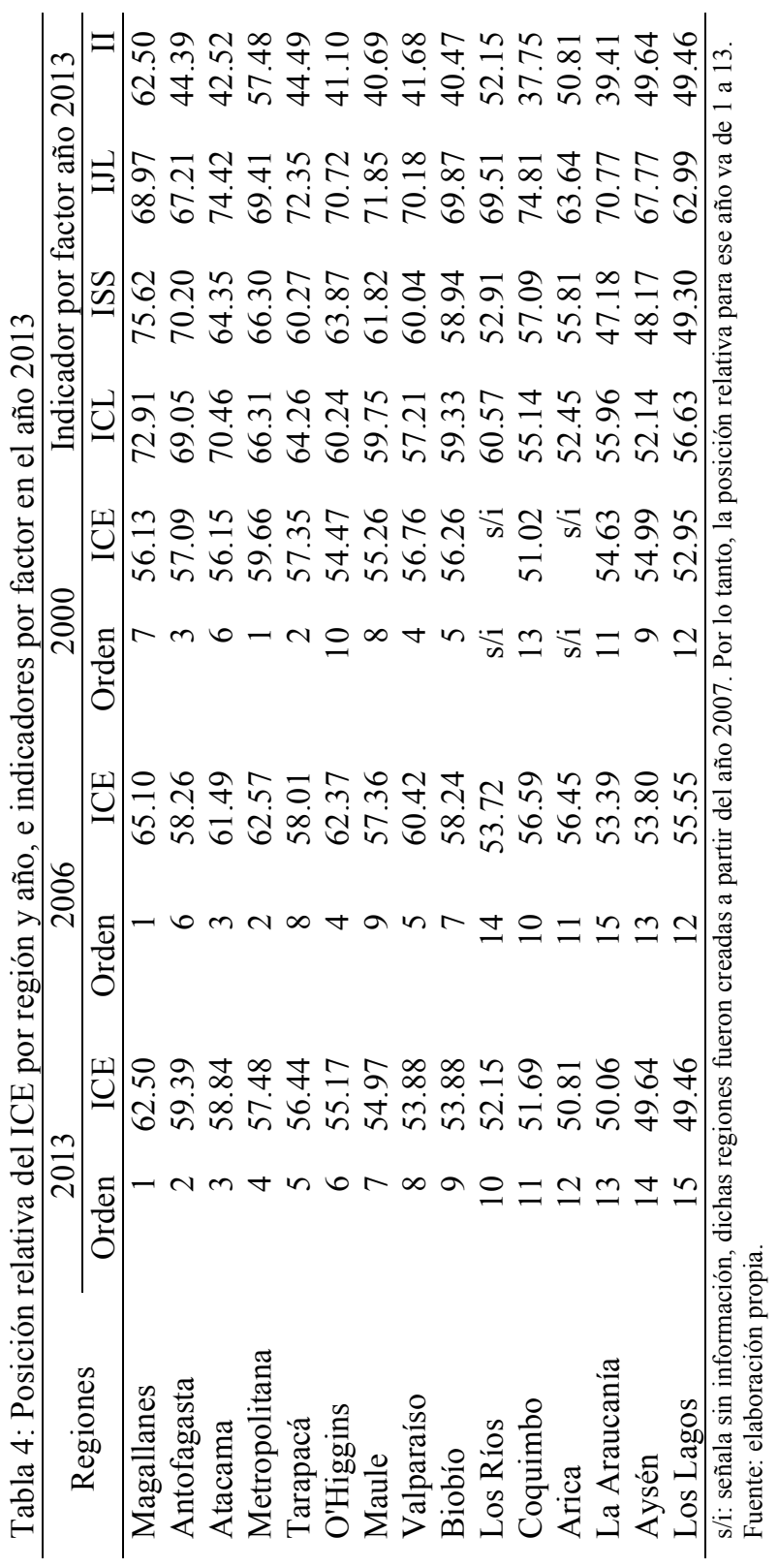


A su vez, las regiones ubicadas en las tres mejores posiciones son las que ostentan una economía con una mayor participación en el sector minería, lo que coincide con ser el sector más dinámico que genera mayores ingresos en la matriz productiva de Chile.

Para 2006 y 2000, se obtiene una posicisión favorable en forma constante para la región Metropolitana y desfavorble para la Araucanía y Los Lagos. Las oscilaciones que tiene la posición relativa de Antofagasta y Atacama probablemente están influídas por que en dichos años se presentan variaciones en el mercado de la minera.

Sin embargo, como se aprecia en la Tabla 3 se obtienen diferencias significativas en todos los índicadores parciales y sintéticos entre las regiones en los tres años.

\section{CE Y SECTORES ECONÓMICOS}

Ahora bien, como se ha señalado, es posible que las diferencias territoriales indicadas anteriormente estén asociadas a los sectores productivos dominantes en la actividad ecónomica de la región. En la Tabla 5 se aprecia que los ocupados en actividades asociadas a suministro de electricidad, gas y agua, minería y servicios financieros, se ubican en mejor posición relativa en cuanto al indicador global de calidad de empleo; éstas últimas dos son precisamente las actividades productivas que tienen mayor participación en las regiones mejor ubicadas en orden de jerarquía señalado en la Tabla 4. Por ejemplo la región Metropolitana se especializa en el sector financiero, y la producción del sector minero se localiza principalmente en las regiones de Antofagasta, Atacama y O'Higgins.

A su vez, el sector pesca y silvoagricultura se jerarquiza en las útimas posiciones; lo que es coincidente con que dichas actividades productivas se localicen principalmente en regiones ubicadas en posición desfavorable: Araucanía, Los Lagos y Aysén. Es interesante constatar que el comercio, hoteles y restaurantes se ubique en útimo lugar en el rankig, en particular al considerar que son actividades intensivas en mano de obra y tienen precísamente la menor calidad de empleo.

Además, se detectan diferencias estadísticas entre los sectores, en los distintos indicadores y años (Tabla 3). Los resultados también revelan, que con excepción del IJL en los años 2013 y 2000, en todas las variables se constatan diferencias significativas en todos los índices. 


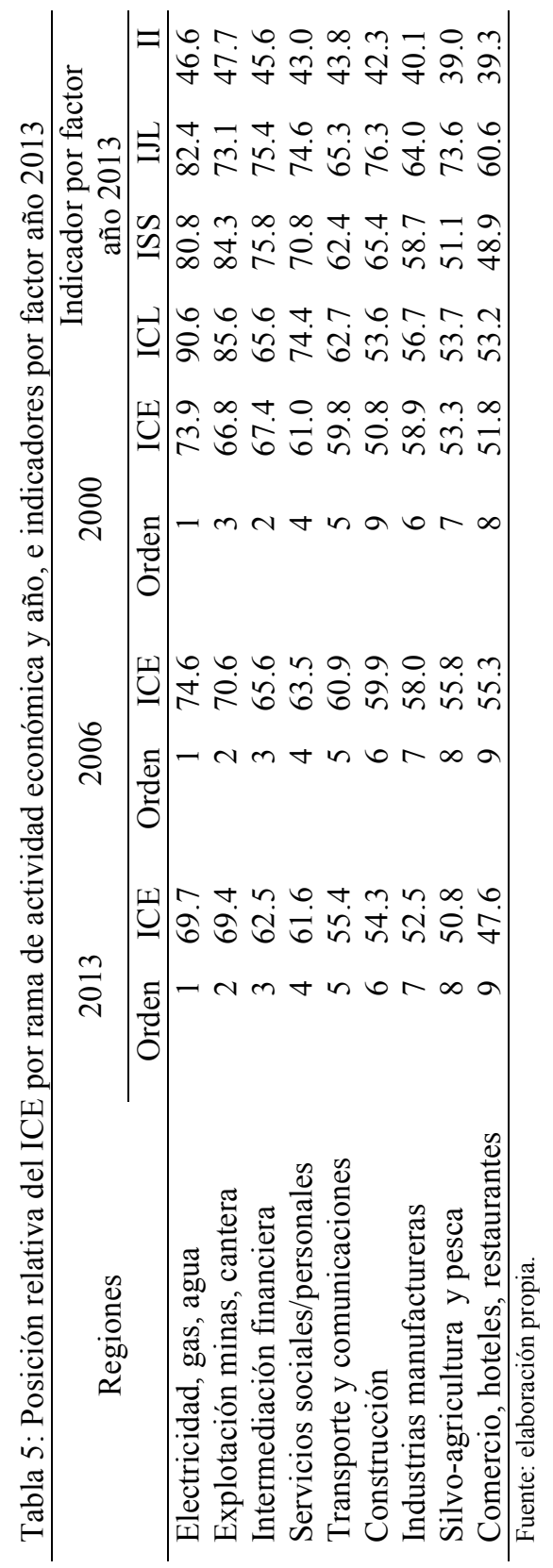




\section{Conclusiones}

Nos enfrentamos a un cambio demográfico acelerado, con bajo crecimiento poblacional y mayor sobrevida de los adultos mayores. A su vez, las políticas públicas dirigidas a este grupo etario se enmarcan en el enfoque y los objetivos suscritos por el país en sucesivos acuerdos internacionales, para el bienestar individual y colectivo de las personas. Este cambio introduce un nuevo paradigma, el envejecimiento activo, el cual deja de identificar la vejez como deterioro, para pasar a ser percibida como una etapa donde es posible el desarrollo de habilidades y destrezas, el crecimiento personal y la elaboración de nuevas metas.

Este nuevo sentido de la vejez y el envejecimiento introduce a su vez una nueva perspectiva, el enfoque de derechos, como la forma de diseñar, implementar y evaluar los planes, programas y proyectos que se implementan desde el Estado, donde los sujetos no son tratados como pasivos sino con derechos inalienables que deben ser respetados y protegidos.

El derecho al trabajo, con los elementos técnicos y humanos que garanticen su realización, conforman la prolongación de esta garantía hacia este grupo etario. El Estado ha de procurar su provisamiento y calidad con dos objetivos principalmente: posibilitar la realización personal de manera que se sientan activas y contribuyan al desarrollo de la sociedad en que viven; y permitir la entrada de ingresos económicos, en el contexto de un aumento de la duración del periodo post-retiro (jubilación) provocado por la extención de la esperanza de vida y posiblemente la falta de ingresos monetarios que permitan solventar los gastos que esto conlleva (Paz, 2010).

En Chile, si bien se ha detectado este fenómeno, aún hay pocos estudios que den cuenta de los efectos que tiene este cambio sociodemográfico. Esta investigación indagó sobre las condiciones laborales en nuestro país de los trabajadores de 60 años o más, con la finalidad de contribuir a la discusión de esta realidad, y a la generación de políticas públicas que permitan el apoyo a su existencia y protección.

El análisis cuantitativo se realiza con base a la propuesta metodológica de Chacón (1999) y Farné (2003), de esta forma se miden ciertos factores de la calidad del empleo, éstos son: Ingreso, Contrato Laboral, Seguridad Social y Jornada Laboral, los que se ponderan para obtener un índicador sintético. Los datos provienen de la Encuesta de Caracterización Socioeconómica Nacional (Casen) 2013, 2006 y 2000.

Se obtienen índicadores sintéticos de calidad de empleo por segmentos. Cada uno de ellos se construye con base a las variables: sexo, zona urbana 
o rural, región, escolaridad, tamaño de la empresa, rama de actividad y edad. Luego se ordenan los grupos de mayor a menor valor del índice. A su vez, se observa la persistencia de la posición relativa de calidad de empleo de cada segmento en los años 2013, 2006 y 2000.

El cálculo de los indicadores nos permite construir un panorama general de la situación laboral de los adultos mayores en Chile. La distancia relativa de la calidad del empleo se da en todos los segmentos analizados, de esta forma los adultos mayores con más altos indicadores de calidad de empleo son los varones, con mayor escolaridad, que residen en el sector urbano y que se encuentran en el tramo de edad entre 60 y 69 años.

Además, en las regiones que desarrollan los polos productivos del país (sector finaciero y mínero), Región Magallanes, Metropolitana, Antofagasta y Atacama, observan mejor calidad de empleo; en comparación a aquellas de economía tradicional (pesca, silvoagricultura), actividades más ligadas a zonas rurales y preferentemente localizadas en las regiones como la Araucanía, Los Lagos y Aysén.

A la vez, en las empresas de mayor tamaño (medianas y grandes), presentan mayor protección a la seguridad social y mejores condiciones contractuales a sus empleados (contrato escrito, indefinido y jornada legal laboral) en comparación a las pequeñas (menores a 50 personas) y a las microempresas (de nueve o menos trabajadores).

Con base en lo anterior, y en busqueda de un envejecimiento activo con mejores condiciones de empleo, y a modo de propuesta se plantea:

- Impulsar una planificación gerontológica en las áreas productivas orientada a facilitar el desempeño laboral de los adultos mayores. Planes y programas de inserción laboral, con financiamiento público y privado, y colaboración profesional a nivel regional y local.

- Incentivar la contratación de adultos mayores mediante flexibilización de sus jornadas laborales y reempazando obligaciones de seguros de salud tradicional (cuando se tengan) por seguros específicos y eliminando el seguro de desempleo obligatorio.

- Fortalecer la educación formal de los adultos mayores, al menos de 12 años de escolaridad, y actualizar competencia con metodologías de enseñanzas adecuadas para personas mayores.

- Apoyar la contratación de adultos mayores en empresas de menor tamaño y el emprendimiento, en particular en sectores poco dinámicos y zonas rurales. 
Todas estas propuestas debiesen incorporar una diferenciación conforme a las caracteristicas del territorio en que residen, aspectos culturales y procesos de desarrollo social que se esten impulsando a nivel local.

\section{REFERENCIAS BIBLIOGRÁFICAS}

Alarcón, R. y Santos, H., 2008, Una mirada longitudinal de la calidad del empleo y sus diferencias desde la perspectiva de género: Encuesta Panel CASEN 19962001-2006, Ministerio de Planificación, Gobierno de Chile, Santiago de Chile.

Bustamante, C. y Arroyo, S., 2008, "La raza como determinante del acceso a un empleo de calidad: un estudio para Cali”, en Ensayos sobre Política Económica, 26(57), pp. 130-175.

Cácerez, D. y Zuñiga, S., 2013, "Disparidades regionales y estabilidad de la calidad del empleo en Chile: 1998-2006", en Gestión y Política Pública, 22(1), pp. 203-243.

Chacón, B., 1999, "Calidad del empleo y pobreza en Chile, 1990-1996, en Infante, R. (ed.), La calidad del empleo, la experiencia de países latinoamericanos y de Estados Unidos, OIT, pp. 181-237, Santiago de Chile.

Contreras, D. y Plaza, G., 2007, Participación laboral femenina en Chile. ¿Cuánto importan los factores culturales? Serie Documentos de Trabajo, Departamento Economía, Universidad de Chile, abril, Issue 235, p. 22.

Farné, S., 2003, Estudios sobre la calidad del empleo en Colombia. Organización Internacional del Trabajo. Oficina Regional para América Latina y el Caribe.

Fernández-Ballesteros, 2012, Sobre envejecimiento activo y participación de los adutos mayores, Organización Iberoaméricana de Seguridad Social, España.

Fernández-García, T. y Ponce de León Romero, L., 2013, "Envejecimiento activo: recomendaciones para la intervención social desde el modelo de gestión de casos", en Portularia, 13(1), pp. 87-97.

Marfán, J., 2007, Adultos mayores en la fuerza del trabajo: inserción en el mercado laboral y determinantes de la participación, Universidad de Chile, Santiago de Chile.

Mora, J. y Ulloa, M., 2011, "Calidad del empleo en la principales ciudades colombianas y endogeneidad de la educación", en Revista de Economía Institucional, 13(25), pp. 163-177.

Ochoa, G., 2011, Germina, consultado el 7/04/2016, disponible en http://germina. $\mathrm{cl} /$ secciones/articulos/politicas-publicas-y-enfoque-de-derechos

Oelckers, F., 2015, "Emprendimiento en la tercera edad: una revisión de la situación actual", en Journal of Technology Management and Innovation, 10(3), pp. 143-153.

OIT, 1999, Trabajo Decente. Ginebra. 
OIT, 2016, Trabajo decente, disponible en http://www.ilo.org/global/topics/decent-work/lang--es/index.htm, consultado el 15/04/2016.

Olivi, A., Fadda-Cori, G., Fadda-Cori, G. y Pizzi-Kirschbaum, 2015, "Evaluación de la calidad de vida de los adultos mayores en la ciudad de Valparaíso", en Papeles de Población, abril-junio, núm. 84, pp. 227-249.

OMS, 2002, "Envejecimiento activo: un marco político", en Rev Esp Geriatr Gerontol 37(S2), pp. 74-105, Organización Mundial de la Salud.

ONU, 1992, Carta de Principios a favor de las Personas Mayores, Conferencia Internacional sobre el Envejecimiento de la Organización de Naciones Unidas, Nueva York.

Paz, J., 2010, Envejecimiento y Empleo en América Latina y el Caribe. Documento de Trabajo, núm. 56, p. 59.

Pontificia Universidad Católica, 2012, Gerontología Social. Capítulo I: Introducción a la Gerontología Social y Conceptos Básicos de Geriatría, Santiago de Chile: RIL Impresores.

Sánchez-González, D. y Egea-Jimenez, C., 2011, "Enfoque de vulnerabilidad social para investigar las desventajas socioambientales. Su aplicación en el estudio de los adultos mayores", en Papeles de Población, julio-diciembre, 17(69), pp. 151-185.

Sehnbruch, K., 2007, "From the quantity to the quality of employment", en The Capability Approach in Human. Berkeley: Cambridge University Press, pp. 1-57.

Sehnbruch, K. y Ruiz-Tagle, J., 2010, The quality of employment: a missing link in development strategies? the example of the chilean labour market, Santiago de Chile.

\section{ResUmen CURRICULAR DE LOS AUTORES}

\section{Luz María Ferrada Bórquez}

Doctora en Economía Aplicada y Análisis Regional, Magister en Economía y Gestión Regional. De profesión Ingeniero Comercial de la Universidad Austral de Chile. Académica de la Universidad de Los Lagos desarrollando investigación y docencia. Directora del Núcleo en Economía Regional. Su línea de investigación es Economía Regional, Mercado Laboral y Calidad de vida. Actualmente participa en diversos proyectos de investigación e innovación. Sus publicaciones más recientes son: "Disparidades regionales de la bancarización en Chile. Periodo 2001-2012”, Aceptada en la Revista Economía Chilena del Banco Central de Chile; “Existe una ventaja de salario para los habitantes de la región de Magallanes?: análisis a partir de un enfoque por género", en Magallania (Punta Arenas), vol. 42, núm. 1, Universidad de Magallanes; "Competitividad y desempeño económico del 
sector silvoagropecuario a nivel regional en Chile", en Idesia, vol. 32 núm. 4. Universidad de Tarapacá; Antipa Camila, Morales Rodrigo y Ferrada Luz María, 2012, "Estimación de funciones de producción en microempresas informales de la ciudad de Osorno Chile", en Estudios Gerenciales, vol. 28 núm. 124, Universidad ICESI; "Diferencias Regionales en la participación laboral de femenina en Chile, en Cuadernos de Economía-Latin American Journal of Economics, vol. 47, Noviembre, Pontificia Universidad Católica de Chile

Dirección electrónica: Iferrada@ulagos.cl

\section{Mauricio Alejandro Ferrada Bórquez}

Licenciado en Trabajo Social, de profesión Asistente Social mención Trabajo Social en Familia y Menores, estudió en la Universidad de los Lagos, y se tituló en el año 2003. Se ha especializado académica y profesionalmente en gerontología, posee diversos pos títulos en la temática, como: el Diplomado en Prevención y Abordaje del Maltrato hacia las Personas Mayores realizado en la Pontificia Universidad Católica de Chile, del Programa del Adulto Mayor y Escuela de Psicología. A su vez, el Diplomado en Gerontología Social también en la Pontificia Universidad Católica de Chile, Facultad de Ciencias Sociales. También desarrolló el Diplomado en Políticas Públicas de Superación de la Pobreza, en la Pontificia Universidad Católica de Chile; y el pos título intervención con Familias en Extrema Pobreza en la Facultad de Ciencias Sociales de la Universidad de Chile. Profesionalmente entre 2005 y 2015 desarrollo funciones en la administración pública coordinando e implementando diversas políticas sociales, con orientación en adultos mayores y familia. Ha sido relator, se destaca la formación para Dirigentes Mayores en las regiones Metropolitana y O'Higgins, a través de Universidad Mayor / U3E y el Servicio Nacional del Adulto Mayor (SENAMA). Hoy ejerce libremente la profesión desarrollando asesorías y capacitación.

Dirección electrónica: mferrada1602@gmail.com 